\title{
4) Pesticide Pneumonitis
}

\author{
Yasutami Kinoshita and Isamu OGIma \\ The Second Department of Internal Medicine, Niigata University \\ School of Medicine, Niigata
}

As most of the pesticides are more or less toxic to man and they are usually sprayed or spinkled to be used, their harmful effect through breathing to respiratory organs should be considered.

Though lesions in skin, mucosa, digestive system and nervous system have been reported as the organs affected by pesticide poisoning, except in case used for suicide, the toxic effect against respiratory system has hardly been reported.

So we will make some points concerning its toxic effect to the lung based on the clinical and the pathological observations which we have done in the last few years.

I) The clinical aspect:

Blasticidin-S (BC-S) is a kind of antibiotics manufactured in our country as the superior blastcides to the mercurious drugs, and is widely used. We experienced the mass outbreak of the poisoning by this drug in summer, 1964.

In addition to the symptoms of fever, headache, diarrhea, skin disease, conjunctivitis and the others, which have been ascribed to the poisoning, many respiratory signs, for example, cough, dyspnea were noticed. The chest X-ray films in some of these cases showed the lung bilaterally with diffuse, slight abnormal shadow.

Studies are based on the observations of 13 patients, additionally to the BC-S cases mentioned above, including cases with old pulmonary tuberculosis which were deteriorated with severe respiratory symptoms after the BC-S inhalation, and cases which showed severe symptoms of chronic pneumonitis after inhalation of herbicides PCP, as well as cases with abnormal pulmonary findings in X-ray after the use of insecticides BHC.

II) The pathological aspect:

In order fo clarify the histological changes in the lungs by above mentioned drugs and to differentiate from common bacterial pneumonia, a group of rabbits were given tracheally with BC-S solution of various concentration and the lungs were investigated serially with light and electron microscope.

We confirmed the pathological pictures quite different from those seen in bacterial pneumonia or farmer's lung in America and Europe and also some interesting findings about injury and regeneration of lung tissue.

From those clinical and experimental observations we would like to present the new concept of pesticide pneumonitis and to alarm its chronic progression. 